\title{
Tunable translational control using site-specific unnatural amino acid incorporation in Escherichia coli
}

Yusuke Kato

Translation of target gene transcripts in Escherichia coli harboring UAG amber stop codons can be switched on by the amber-codon-specific incorporation of an exogenously supplied unnatural amino acid, 3-iodo---tyrosine. Here, we report that this translational switch can control the translational efficiency at any intermediate magnitude by adjustment of the 3iodo--tyrosine concentration in the medium, as a tunable translational controller. The translational efficiency of a target gene reached maximum levels with $10^{-5} \mathrm{M}$ 3-iodo-tyrosine, and intermediate levels were observed with suboptimal concentrations (approximately spanning a $2-\log _{10}$ concentration range, $10^{-7}$ to $10^{-5} \mathrm{M}$ ). Such intermediatelevel expression was also confirmed in individual bacteria. 
2 Author(s):

3 Yusuke Kato

4

5 Affiliation \& Location:

6 Genetically Modified Organism Research Center,

7 National Institute of Agrobiological Sciences,

8 Tsukuba, Ibaraki, Japan.

9

10 *Address for correspondence:

11 Yusuke Kato, Ph.D.

12 Genetically Modified Organism Research Center

13 National Institute of Agrobiological Sciences

14 Oowashi 1-2

15 Tsukuba, Ibaraki, Japan

16 Phone/Fax: +81-29-838-6059

17 Email: kato@affrc.go.jp 


\section{Abstract}

19

20 Translation of target gene transcripts in Escherichia coli harboring UAG amber stop codons can be

21 switched on by the amber-codon-specific incorporation of an exogenously supplied unnatural amino

22 acid, 3-iodo- - -tyrosine. Here, we report that this translational switch can control the translational

23 efficiency at any intermediate magnitude by adjustment of the 3-iodo- ${ }_{\mathrm{L}}$-tyrosine concentration in the

24 medium, as a tunable translational controller. The translational efficiency of a target gene reached

25 maximum levels with $10^{-5} \mathrm{M}$ 3-iodo-L-tyrosine, and intermediate levels were observed with suboptimal

26 concentrations (approximately spanning a $2-\log _{10}$ concentration range, $10^{-7}$ to $10^{-5} \mathrm{M}$ ). Such

27 intermediate-level expression was also confirmed in individual bacteria. 


\section{Introduction}

30 Controlling gene expression is a key methodology for biotechnology. Particularly, its fine-tuning and

31 optimization are important for construction of artificial gene circuits in synthetic biology and for

32 metabolic engineering. Tunable conditional expression systems regulated by extracellular

33 inducers/repressors are thus very useful.

We previously demonstrated a translational switch using site-specific unnatural amino acid

35 (UAA) 3-iodo- ${ }_{\mathrm{L}}$-tyrosine (IY) incorporation in natural amber suppressor-free strains of Escherichia

36 coli (Minaba and Kato, 2014). Although IY is not perfectly "unnatural" and found in very specific

37 biological tissues such as the thyroid cells (Tietze et al., 1989) and the sponge skeletons (Ueberlein et

38 al., 2014), we cannot detect IY ubiquitously in natural environments. The translational switch is based

39 on conditional read-through of the UAG amber stop codons that are inserted in target genes (Fig. 1). A

40 variant of aminoacyl-tRNA synthetase (aaRS) IYRS that was derived from the archaebacterium

41 Methanocaldococcus jannaschii specifically recognizes both IY and an amber suppressor tRNA

42 (tRNA $\left._{\text {CUA }}\right)$ MJR1 (Sakamoto et al., 2009). Extracellular IY is taken up and incorporated into proteins

43 at sites of the amber codons in the IYRS/ MJR1-expressing cells. The target gene transcripts with the

44 amber stop codons inserted next to the AUG translational start codon are translated only in the

45 presence of IY. The absence of IY prevents translation of the target genes. 

and Kato, 2014). First, this translational switch also can be controlled by the induction/repression of either aaRS or $\mathrm{tRNA}_{\mathrm{CUA}}$, in addition to the presence/absence of UAA. The switchability can be

49 modulated by combination use with aaRS- and/or tRNA $\mathrm{CUA}^{-}$switching. Second, UAA does not naturally exist. Third, UAA is a "building block" that forms an aminoacyl-tRNA and target proteins,

51 distinct from simple regulation-specific molecules, such as isopropyl $\beta{ }^{-}{ }^{-1-t h i o g a l a c t o p y r a n o s i d e ~}$ (IPTG) for the derepression of the lactose operator. The switching mechanism involves the direct

53 incorporation of UAA into target proteins, and is not effective for interventional systems. The second and third characteristics indicate that the UAA-controlling translational switch is robust against environmental and host-endogenous noises. Although an inducible $\mathrm{tRNA}_{\mathrm{CUA}}$ has been used as a similar translational switch based on amber suppression, these features are not found (Zengel and Lindahl, 1981; Herring et al., 2003). Fourth, the UAA-controlling translational switch can be used in combination with any established transcriptional switches to obtain a synergistic regulatory effect. Using this strategy, we constructed a high-yield and zero-leakage expression system, in which strong expression by the $\mathrm{T} 7$ promoter was maintained under the induction condition, and almost no proteins were expressed under the repression condition by double control of transcription-translation. Riboswitches and small regulatory RNAs (sRNAs) are well known as post-transcriptional controlling tools in E. coli. Some riboswitches that are located in non-coding portions of mRNAs can 
64 regulate gene expression in cis by binding a specific small molecule via controlling translational

65 initiation and/or mRNA degradation (Caron et al., 2012). The sRNAs are trans-acting and target gene

66 alterations are not required, although off-target effects are often detected (Bobrovskyy and Vanderpool,

67 2013). The sRNAs modulate the translation via controlling translation initiation and/or mRNA

68 degradation. The distinct characteristics of these RNA-based post-transcriptional switches suggest that

69 the UAA-controlling translational switch is complementary rather than competitive with the others. To date, many systems for the incorporation of various UAAs have been developed (Liu and

71 Schultz, 2010). In addition, similar translational switches controlled by UAAs are expected to be

72 applicable also for eukaryotes, such as yeasts, nematodes, insects, mammalian cells, and plants,

73 because the site-specific unnatural amino acid incorporation systems have already been introduced

74 (Chin et al., 2003; Greiss and Chin, 2011; Bianco et al., 2012; Sakamoto et al., 2002; Li et al., 2013). The target gene products that are controlled by the UAA-controlling translational switch

76 necessitate the incorporation of UAA. Although the UAA-incorporation may cause functional

77 alterations in some target proteins, we can avoid this problem by neutral site selection, incorporation

78 into tag or processed-out sequences. Alternatively, the UAAs can be used as tools to facilitate

79 purification or tracking of target gene products (Minaba and Kato, 2014).

81 switch. Here, we studied the intermediate states between the fully on- and off-states of the translational 
82 switch. The results suggest that this switch can control the translational efficiency at any intermediate

83 magnitude by adjustment of the appropriate IY concentration, and thus, function as a tunable

84 translational controller. 
85

86

87

88

89

90

91

92

93

94

95

96

97

98

99

100

101

102

\section{Materials and Methods}

列

Fluorescence measurement of pooled bacteria

Assays were performed as described previously (Minaba and Kato, 2014). Briefly, we used E. coli

BL21-AI [ $F^{-}$ompT gal dcm lon $h s d S_{B}\left(r_{B}^{-} m_{B}^{-}\right)$araB::T7RNAP-tetA] carrying the plasmid pTYR

MjIYRS2-1(D286) MJR1×3 encoding IYRS and MJR1 and the amber-inserted EGFP expression

plasmid (driven by the E. coli lpp promoter with an amber stop codon TAG inserted next to the start

codon ATG). The sequence for the EGFP expression plasmid is shown in Fig. S1. An overnight

(approximately $16 \mathrm{~h}$ ) culture of bacteria was resuspended in an equal volume of liquid LB medium

4 ( $1 \%$ bacto tryptone, $0.5 \%$ yeast extract, and $1 \% \mathrm{NaCl})$ containing various concentrations of optically-

5 pure IY. IY was directly dissolved and diluted in LB medium. After a $6 \mathrm{~h}$ culture $(2 \mathrm{ml}$ in a $14 \mathrm{ml}-$

6 culture tube at $37^{\circ} \mathrm{C}$ with rotary shaking at $200 \mathrm{rpm}$ ), the bacteria were collected by centrifugation

(1,800 $\times g$ for $3 \mathrm{~min})$. The pellet was washed and was resuspended in an equal volume of $0.9 \%(\mathrm{wt} / \mathrm{vol})$

$8 \mathrm{NaCl}$, and this wash step was repeated twice. An aliquot of the bacterial suspension $(150 \mu \mathrm{l})$ was

9 diluted in $3.0 \mathrm{ml}$ of the $0.9 \% \mathrm{NaCl}$, and the $\mathrm{OD}_{590}$ was measured. The fluorescence intensity of the bacterial population was measured using a Shimadzu RF-5300PC spectrofluorometer (excitation, 480

$\mathrm{nm}$; emission, $515 \mathrm{~nm})$. The background fluorescence was estimated using the bacteria carrying a

MJR1-deleted plasmid ( $\triangle \mathrm{MJR} 1)$ at IY=0 (no significant IY-dependency was observed, Fig. S2). The 
103 background-subtracted values were used to calculate the points for the dose-response curve. For the

104 time course measurement of EGFP accumulation, we cultured the bacteria in $20 \mathrm{ml} \mathrm{LB}$ medium in a

$105200 \mathrm{ml}$ culture flask. Aliquots of the bacterial culture $(150 \mu \mathrm{l})$ were withdrawn at various time points,

106 and the $\mathrm{OD}_{590}$ and fluorescence were measured.

107

108 Photomicrographs

109 Photomicrographs and Nomarski differential interference contrast images of the fluorescent bacteria

110 were recorded using both a Carl Zeiss Axioskop 2 with a 38-HE Endow GFP filter-set and a Roper

111 Scientific Photometrics CoolSNAP ver.1.1.

112

113 Image analyses

114 Analyses of the fluorescence images were performed using ImageJ 1.48v (National Institutes of Health,

115 U.S.A.). Prior to analyses, the fluorescence images were confirmed as not being saturated at any pixels.

116 Three-dimensional graphs of the intensities of the pixels were generated using Surface Plot command.

117 The fluorescence intensity of individual bacteria was measured using Particle Analysis command. A

118 background value (bacteria-absent area) was used as a threshold for particle detection. A range of

119 particle area was set to detect only individual and not-overlapping bacterial cell images. 
121 Growth curve

122 Growth curves were determined as previously described with some modifications (Minaba and Kato,

123 2014). Overnight cultures of bacteria were diluted (1:200) in fresh LB medium and were incubated at

$12437^{\circ} \mathrm{C}$ with rotary shaking at $200 \mathrm{rpm}$. After reaching a visible density (around $\mathrm{OD}_{590}=0.1$ ), $\mathrm{OD}_{590}$ was

125 measured every $20 \mathrm{~min}$ for $2 \mathrm{~h}$. 


\section{Results and Discussion}

128 To characterize the intermediate states of the translational switch using the IY-incorporation system,

129 the IY dose-dependency of translational efficiency for a target gene was first determined for a bacterial

130 population (Fig. 2A). As shown in Fig. 1, an EGFP gene containing an amber stop codon next to the

131 ATG translational start codon was constitutively transcribed by the lpp promoter in the E. coli BL21-

132 AI strain expressing IYRS and MJR1. The translational efficiency was estimated from the EGFP

133 fluorescence $6 \mathrm{~h}$ after IY addition into the medium. The "gross" translational efficiency for the

134 population started to increase significantly at $3 \times 10^{-7} \mathrm{M}$ and reached maximum levels at $10^{-5} \mathrm{M}$.

135 Intermediate levels of translational efficiency were observed at a $2-\log _{10}$ suboptimal concentration of

136 IY $\left(10^{-7}\right.$ to $\left.10^{-5} \mathrm{M}\right)$, suggesting that the translational efficiency can be tuned in this concentration range.

137 The $50 \%$ effective concentration was estimated to be $3 \times 10^{-6} \mathrm{M}$. The IY concentration-dependent

138 change of the gross translational efficiency was also confirmed by a direct measurement of the time

139 course for EGFP accumulation (Fig. 2B). The EGFP accumulation rate was clearly slower at

140 suboptimal concentrations than that at the optimal concentration. The accumulation rate increased with

141 increasing IY concentration, also suggesting that an intermediate translational efficiency could be

142 obtained in the suboptimal concentration range. 
144 an individual bacterium. In the case of conditional gene expression by the araBAD promoter,

145 intermediate expression levels in the cultures reflected a population average of the induced and non-

146 induced cells, i.e., each cell responded to an inducer ${ }_{\mathrm{L}}$-arabinose at a suboptimal concentration in an

147 all-or-none manner (Siegele and Hu, 1997; Guzman et al., 1995). A similar non-uniform induction was

148 also reported for the lac operon (Novick and Weiner, 1957; Maloney and Rotman, 1973). We therefore

149 determined whether the IY dose-dependent change of EGFP fluorescence in individual cells using

150 fluorescence images (Fig. 3). The fluorescence intensity of these images was quantified by image

151 analysis, and spatial maps were generated. The fluorescence intensity clearly increased with increasing

152 IY concentration. Fluorescent intensity distribution histograms of individual bacteria were also

153 examined. As expected from the fluorescence images and their spatial maps, the peak frequency at a

154 suboptimal IY concentration $\left(1 \times 10^{-6} \mathrm{M}\right)$ was located between those at zero and the saturation

155 concentration, indicating that the translational efficiency of individual bacteria can be controlled at

156 intermediate values. This response is unlike the mix-population of all-or-none responding cells

157 observed in a suboptimal concentration of inducers for both the araBAD promoter and the lac operon.

158 The frequency distribution was relatively wide and overlapped with those at zero and at the saturation

159 concentration, suggesting that the responses of individual cells are variable at suboptimal

160 concentrations. The all-or-none behavior of the lac operon was explained by an autocatalytic positive

161 feedback loop that led to a burst of synthesis of galactoside permease LacY (Novick and Weiner, 1957; 
162 Maloney and Rotman, 1973). A similar model was also proposed for the araBAD promoter system

163 (Siegele and $\mathrm{Hu}, 1997)$. In these models, the intracellular concentration of either lactose or arabinose

164 tends to be either very low or saturated. To obtain a better linear response to an extracellular inducer

165 concentration, IPTG is used instead of lactose in a lacY- strain for control of the lac promoter

166 (Khlebnikov and Keasling, 2002). Similarly, mutant strains in which arabinose transport and

167 degradation genes are deficient can avoid the all-or-none response of the araBAD promoter (Bowers et

168 al., 2004). These modifications impair the positive feedback loop and confer linearity between

169 extracellular and intracellular inducer concentrations. In the case of the IY-controlling translational

170 switch, the velocity of translation by a suppressor IY-tRNA is expected to depend on the intracellular

171 IY concentration if the concentrations of the suppressor tRNA, IYRS, and peptide release factors are

172 constant (Yarus et al., 1986). The intracellular IY concentration was possibly at a subsaturation level at

173 the suboptimal concentration of extracellular IY. Although the uptake mechanism of IY remains

174 unclear, positive feedback loops as seen in the lac operon or the araBAD system may be weak or not

175 be involved (Pittard, 1996).

176 Approximately $7 \%$ of the maximum translation was detected even in the absence of IY, as

177 also described previously (Minaba and Kato, 2014). The leaky translation was completely abolished by

178 deletion of the MJR1 gene, suggesting that mischarges of MJR1 were the cause (Fig. 4A and 4B).

179 Although some countermeasure techniques have been proposed, reduction of leaky translation needs to 
180 be a priority (Minaba and Kato, 2014).

181 The translational efficiency decreased from the saturation level at an extremely high IY

182 concentration (Fig. 2A and 2B). Although its mechanism remains to be elucidated, the decrease may

183 not be due to non-specific toxicity of IY because the bacterial growth rates did not decrease both in the

184 IY-incorporating strains and in the parent strain (Fig. S3).

185 In this study, we evaluated the IY-controlling translational switch using a single setting (a

186 single amber codon in one position, and the single target gene EGFP). In the future, further studies

187 using this application should clarify how general this system is. 


\section{Conclusions}

189

190 The translational switch using site-specific IY incorporation can be used as a "tunable translational

191 controller" that regulates the translational efficiency in each individual cell. Using this controller, we

192 expect to be able regulate the translational efficiency over a wide range in combination with any

193 transcriptional controlling systems (Minaba and Kato, 2014). The tunable translational controller is a

194 promising tool for conditional fine-tuning and for optimizing the construction of artificial gene circuits

195 in synthetic biology and in metabolic engineering (Yadav et al., 2012). 


\section{Acknowledgments}

197

198 We thank Kensaku Sakamoto and Shigeyuki Yokoyama (RIKEN) for the IYRS-MJR1

199 expression plasmids and Michael Yarus (University of Colorado) for the luxB gene. 
REFERENCES

Bianco, A., Townsley, F. M., Greiss, S., Lang, K., Chin, J. W. 2012. Expanding the genetic code of Drosophila melanogaster. Nat. Chem. Biol. 8, 748-750.

Bobrovskyy, M., Vanderpool, C. K. 2013. Regulation of bacterial metabolism by small RNAs using diverse mechanisms. Annu. Rev. Genet. 47, 209-232.

Bowers, L. M., LaPoint, K., Anthony, L., Pluciennik, A., Filutowicz, M. 2004. Bacterial expression system with tightly regulated gene expression and plasmid copy number. Gene 340, 11-18.

Caron, M. P., Bastet, L., Lussier, A., Simoneau-Roy, M., Massé, E, Lafontaine, D. A. 2012. Dualacting riboswitch control of translation initiation and mRNA decay. Proc. Natl. Acad. Sci. U. S. A. 109, E3444-E3453.

Chin, J. W., Cropp, A., Anderson, C., Mukherji, M., Zhang, Z., Schulz, P. G. 2003. An expanded eukaryotic genetic code. Science 301, 964-967.

Greiss, S., Chin, J. W. 2011. Expanding the genetic code of an animal. J. Am. Chem. Soc. 133, 14196-14199.

Guzman, L. M., Belin, D., Carson, M. J., Beckwith, J. 1995. Tight regulation, modulation, and highlevel expression by vectors containing the arabinose $P_{B A D}$ promoter. J. Bacteriol. 177(14), 41214130. 
218 Herring, C. D., Glasner, J. D., Blattner, F. R. 2003. Gene replacement without selection: regulated suppression of amber mutations in Escherichia coli. Gene 331, 153-163.

Khlebnikov, A., Keasling, J. D. 2002. Effect of lacY expression on homogeneity of induction from the $\mathrm{P}(\operatorname{tac})$ and $\mathrm{P}(\operatorname{trc})$ promoters by natural and synthetic inducers. Biotechnol. Prog. 18, 672-674.

Li, F., Zhang, H., Sun, Y., Pan, Y., Zhou, J., Wang, J. 2013. Expanding the genetic code for photoclick chemistry in E. coli, mammalian cells, and A. thaliana. Angew. Chem. Int. Ed. Engl. 52, 9700-9704.

Liu, C. C., Schultz, P. G. 2010. Adding new chemistries to the genetic code. Annu. Rev. Biochem. 79, 413-444.

Maloney, P. C., Rotman, B. 1973. Distribution of suboptimally induced $\beta-{ }^{-}$-Galactosidase in Escherichia coli: the enzyme content of individual cells. J. Mol. Biol. 73(1), 77-91.

Minaba, M., Kato, Y. 2014. High-yield, zero-leakage expression system with a translational switch using site-specific unnatural amino acid incorporation. Appl. Environ. Microbiol. 80(5), 17181725.

Novick, A., Weiner, M. 1957. Enzyme induction as an all-or-none phenomenon. Proc. Natl. Acad. Sci. U. S. A. 43(7), 553-566. 
Umbarger, H. E. (Eds.), Escherichia coli and Salmonella: cellular and molecular biology, $2^{\text {nd }}$ ed. ASM press, Washington DC, pp. 458-484.

Sakamoto, K., Hayashi, A., Sakamoto, A., Kiga, D., Nakayama, H., Soma, A., Kobayashi, T.,

Kitabatake, M., Takio, K., Saito, K., Shirouzu, M., Hirao, I, Yokoyama, S. 2002. Site-specific $4692-4699$.

Sakamoto, K., Murayama, K., Oki, K., Iraha, F., Kato-Murayama, M., Takahashi, M., Ohtake, incorporation of an unnatural amino acid into proteins in mammalian cells. Nucleic Acids Res. 30, nonsense suppression. J. Bacteriol. 172(2), 595-602.

Siegele, D. A., Hu, J. C. 1997. Gene expression from plasmids containing the araBAD promoter at 94(15), 8168-8172.

Tietze, F., Kohn, L. D., Kohn, A. D., Bernardini, I., Andersson, H. C., Adamson, M. D., Harper, subsaturating inducer concentrations represents mixed populations. Proc. Natl. Acad. Sci. U. S. A.

lysosomes. J. Biol. Chem. 264, 4762-4765. 
254 Ueberlein, S., Machill, S., Niemann, H., Proksch, P., Brunner, E. 2014. The skeletal amino acid 255 composition of the marine demosponge Aplysina cavernicola. Mar. Drugs 12, 4417-4438.

256 Yadav, V. G., Mey, M. D., Lim, C. G., Ajikumar, P. K., Stephanopoulos, G. 2012. The future of 257 metabolic engineering and synthetic biology: towards a systematic practice. Metab. Eng. 14(3), $258 \quad 233-241$.

259 Yarus, M., Cline, S. W., Wier, P., Breeden, L., Thompson, R. C. 1986. Actions of the anticodon 260 arm in translation on the phenotypes of RNA Mutants. J. Mol. Biol. 192, 235-255.

261 Zengel, J. M., Lindahl, L. 1981. High-efficiency, temperature-sensitive suppression of amber 262 mutations in Escherichia coli. J. Bacteriol. 145, 459-465. 


\section{FIGURE LEGENDS}

264

265 Fig. 1. Schematic of the translational switch using the amber codon-specific IY. An amber stop codon

266 is inserted next to the ATG translational start codon in the target gene (egfp). MJR1 is an amber

267 suppressor tRNA. IYRS is an aminoacyl-tRNA synthetase that orthogonally recognizes IY and MJR1.

268 Extracellular IY is taken up by the bacteria. The addition of IY in the media results in amber stop

269 codon read-through and translation of the target gene. Translation is interrupted in the absence of IY.

270 RF1, peptide chain release factor 1.

271

272 Fig. 2. IY dose-dependent change in translational efficiency for a bacterial population. The

273 experimental system is schematically shown in Fig 1. (A) Dose-response curve. Bacteria were cultured

274 in media containing various concentrations of IY for 6 h. Colored circles indicate the samples for Fig. 3.

275 Data are shown as mean \pm SEM. $n=3$ independent experiments using completely separated bacterial

276 cultures. Statistical analysis was performed using Welch's $t$-test in Excel ver. 14.0. A single fitted

277 dose-response curve (log-logistic, IY $=0$ to $3 \square 10^{-4} \mathrm{M}$ ) was generated using Origin7. The equation

278 for the fitted curve and the assessment of goodness-of-fit are represented under the graph. F,

279 fluorescent intensity (arbitrary unit); $F_{\max }$, initial $\mathrm{F}$ value (right horizontal asymptote); $\mathrm{F}_{\min }$, final $\mathrm{F}$

280 value (left horizontal asymptote); $\mathrm{C}_{\mathrm{IY}}, \mathrm{IY}$ concentration $(\mathrm{M}) ; \mathrm{C}_{0}, 50 \%$ effective $\mathrm{C}_{\mathrm{IY}} ; \chi^{2}$, reduced chi- 
281 squared value; DoF, degrees of freedom. (B) Time course of EGFP accumulation.

283 Fig. 3. EGFP expression in individual bacteria. The sampling points are indicated in the dose-response

284 curve in Fig 2A. Upper and lower photographs are epifluorescence images and Nomarski differential 285 interference contrast images, respectively. The photographic conditions were constant for all of the 286 fluorescence images. Calibration bar $=100 \mu \mathrm{m}$. Asterisks in spatial distribution graphs indicate the 287 upper right corners of the fluorescence images. The frequency in the histograms indicates the number 288 of individual bacteria.

Fig. 4. Leaky translation in the absence of IY. (A) Leaky translation for a bacterial population. Note 291 that the measured fluorescence contains both EGFP-fluorescence and non-EGFP background. 292 Complete set, the strain carrying the plasmid pTYR MjIYRS2-1(D286) MJR1×3 and the amber293 inserted EGFP expression plasmid (driven by the E. coli lpp promoter); $\Delta \mathrm{MJR} 1$, a derivative strain carrying a MJR1-deleted plasmid; $\triangle \mathrm{EGFP}$, a derivative strain lacking the amber-inserted EGFP gene expression plasmid; $\triangle \mathrm{EGFP}+$ Lux, a derivative strain in which the amber-inserted EGFP gene was 296 substituted with an amber-inserted $\operatorname{LuxB}$ gene from the bacterium Vibrio harveyi as a vector control 297 (Shultz and Yarus, 1990). Data are shown as mean \pm SEM. $n=3$ independent experiments using completely separated bacterial cultures. Statistical analysis was performed using Welch's $t$-test. ns, not 
299 significant. (B) Observation of leaky translation in individual bacteria. The EGFP fluorescence was

300 measured in the absence of IY. In these images, non-specific background fluorescence was completely

301 filtered out (note that this method is distinct from that of Fig. 4A). The exposure time for the EGFP

302 images was twice that in Fig. 3. 


\section{1}

Figure 1: Schematic of the translational switch using the amber codon-specific IY.

An amber stop codon is inserted next to the ATG translational start codon in the target gene (egfp). MJR1 is an amber suppressor tRNA. IYRS is an aminoacyl-tRNA synthetase that orthogonally recognizes IY and MJR1. Extracellular IY is taken up by the bacteria. The addition of IY in the media results in amber stop codon read-through and translation of the target gene. Translation is interrupted in the absence of IY. RF1, peptide chain release factor 1.

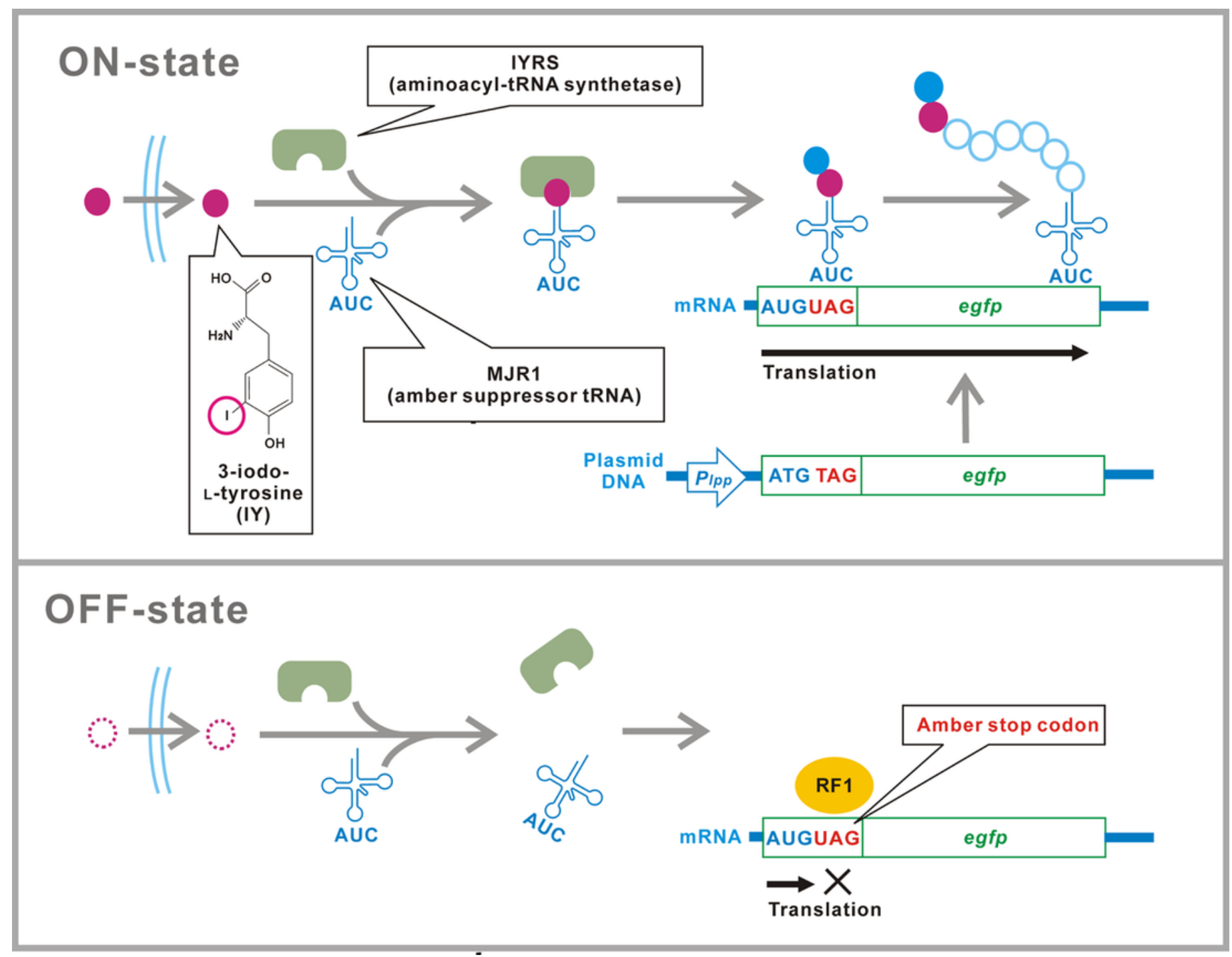


2

Figure 2: IY dose-dependent change in translational efficiency for a bacterial population.

The experimental system is schematically shown in Fig 1. (A) Dose-response curve. Bacteria were cultured in media containing various concentrations of IY for $6 \mathrm{~h}$. Colored circles indicate the samples for Fig. 3. Data are shown as mean $\square$ SEM. $n=3$ independent experiments using completely separated bacterial cultures. Statistical analysis was performed using Welch's t-test in Excel ver. 14.0. A single fitted dose-response curve (loglogistic, $I Y=0$ to $3 \square 10 \mathrm{M}$ ) was generated using Origin7. The equation for the fitted curve and assessments of goodness-of-fit are represented under the graph. F, fluorescent intensity (arbitrary unit); $F_{\text {max }}$ the initial $F$ value (right horizontal asymptote); $F_{\text {min, }}$ the final $F$ value (left horizontal asymptote); $C_{I Y}, I Y$ concentration (M); $C_{0}, 50 \%$ effective $C_{Y Y}$ (point of inflection); $\chi^{2}$, reduced chi-squared value; DoF, degrees of freedom. (B) Time course of EGFP accumulation. 


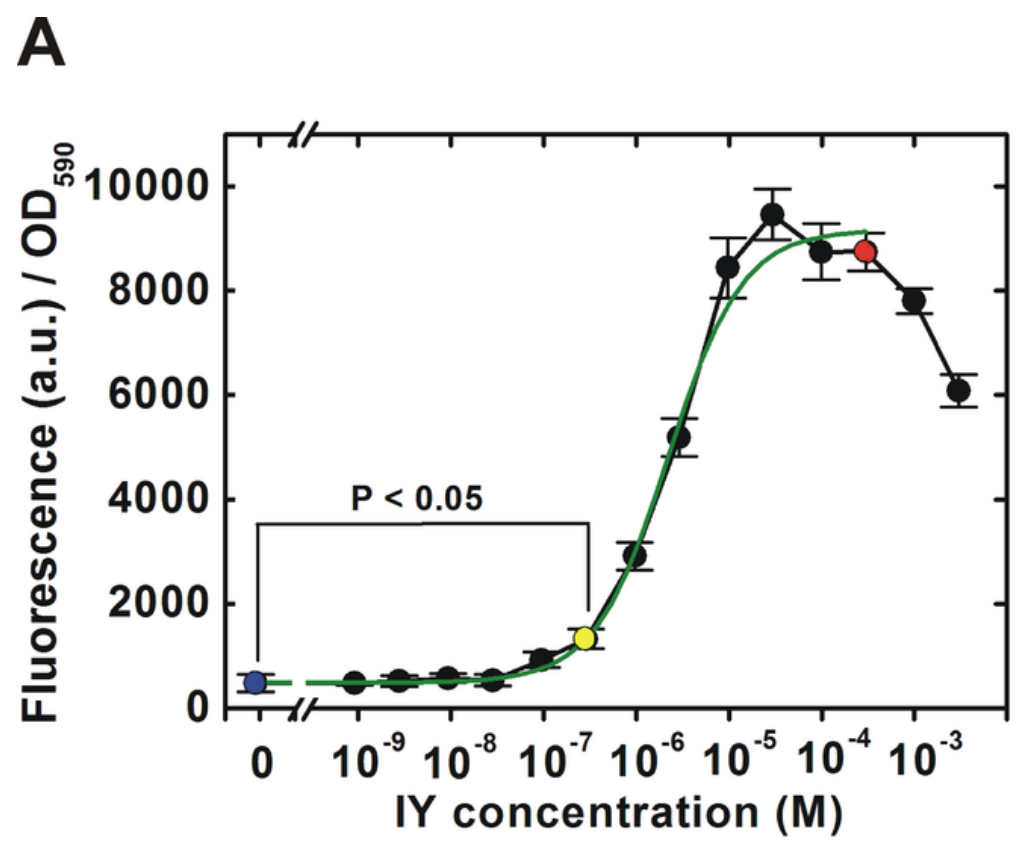

Model: Logistic

$$
\begin{aligned}
F=F_{\text {max }}+\frac{F_{\text {min }}-F_{\text {max }}}{1+\left(C_{\text {IY }} / C_{0}\right)^{p}} \\
F_{\text {max }}=(9.15 \pm 0.29) \times 10^{3} \\
F_{\text {min }}=(0.61 \pm 0.03) \times 10^{3} \\
C_{0}=(2.30 \pm 0.31) \times 10^{-6} \mathrm{M} \\
\text { p }=1.10 \pm 0.11
\end{aligned}
$$

Goodness-of-fit:

$$
\begin{aligned}
\chi^{2} / \mathrm{DoF} & =0.93 \\
\mathbf{R}^{2} & =0.99
\end{aligned}
$$

B

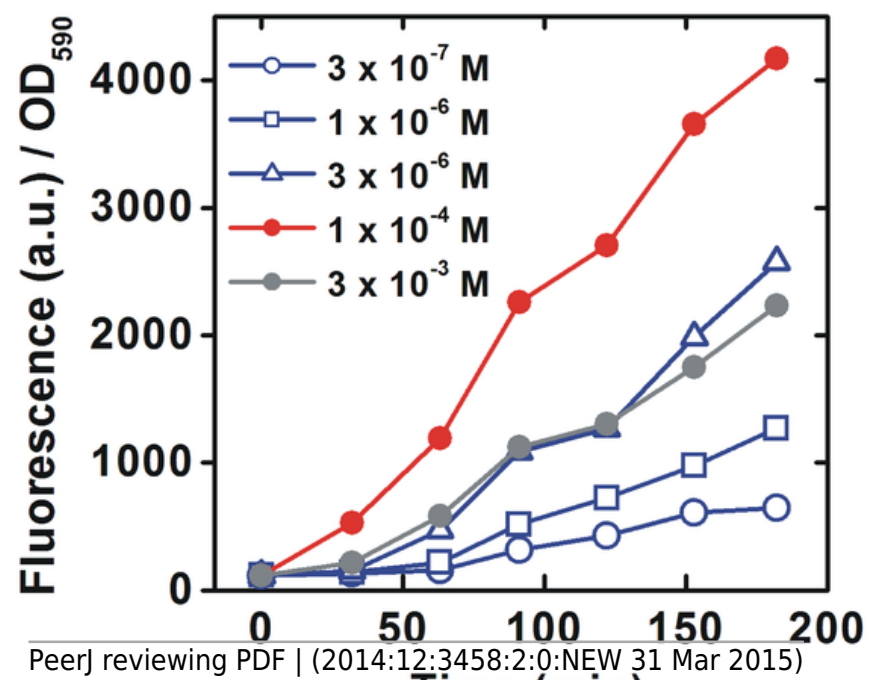




\section{3}

Figure 3: EGFP expression in individual bacteria.

The sampling points are indicated in the dose-response curve in Fig 2A. Upper and lower photographs are epifluorescence images and Nomarski differential interference contrast images, respectively. The photographic conditions were constant for all of the fluorescence images. Calibration bar $=100 \mu \mathrm{m}$. Asterisks in spatial distribution graphs indicate the upper right corners of the fluorescence images. The frequency in the histograms indicates the number of individual bacteria. 


\section{IY concentration (M)}

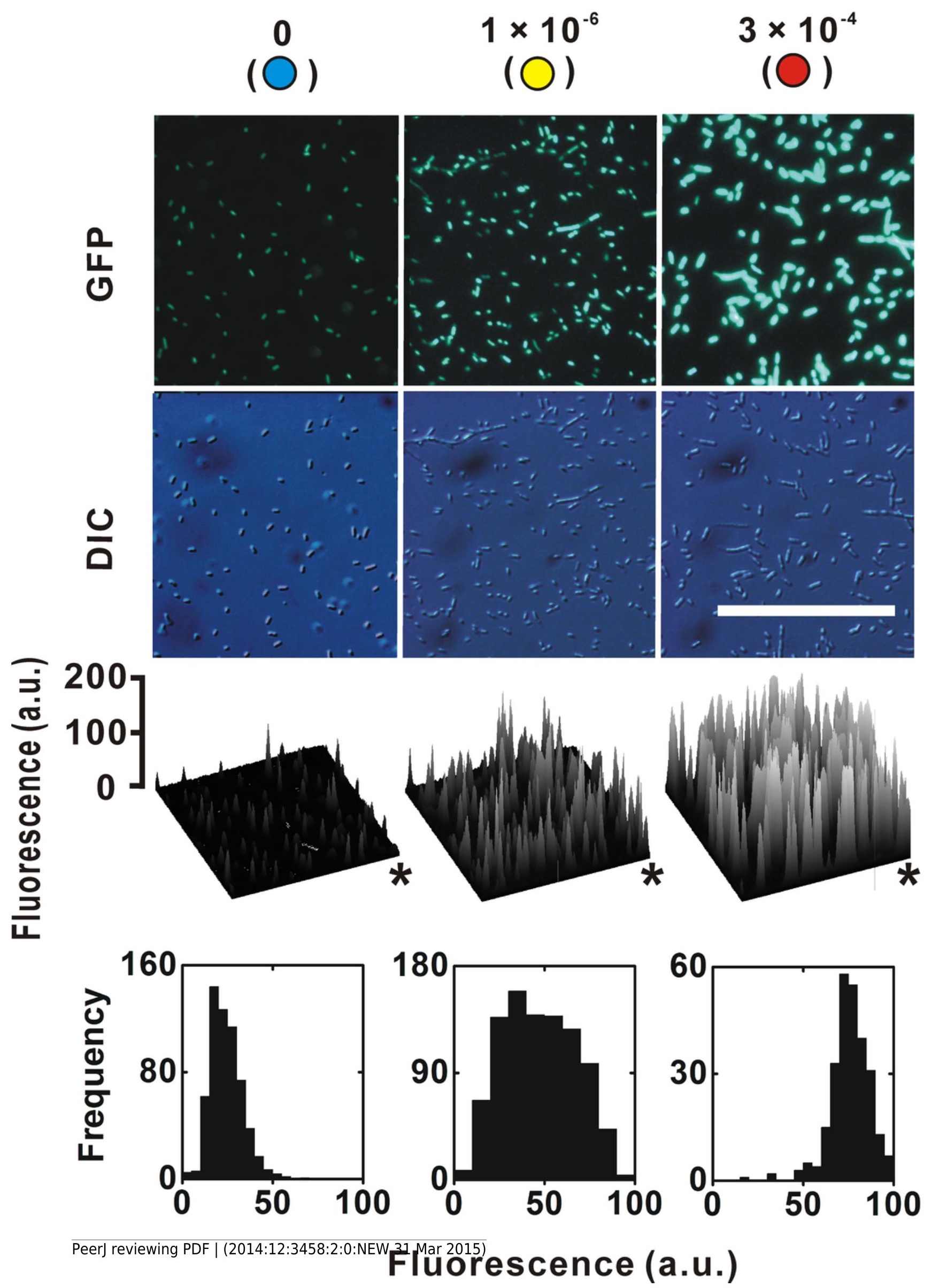




\section{4}

Figure 4: Leaky translation in the absence of IY.

(A) Leaky translation for a bacterial population. Note that the measured fluorescence contains both EGFP-fluorescence and non-EGFP background. Complete set, the strain carrying the plasmid pTYR MjIYRS2-1(D286) MJR1 3 and the amber-inserted EGFP expression plasmid (driven by the E. coli Ipp promoter); $\triangle \mathrm{MJR} 1$, a derivative strain carrying a MJR1deleted plasmid; $\triangle E G F P$, a derivative strain lacking the amber-inserted EGFP gene expression plasmid; $\triangle E G F P+L u x$, a derivative strain in which the amber-inserted EGFP gene was substituted with an amber-inserted LuxB gene from the bacterium Vibrio harveyi as a vector control (Shultz and Yarus, 1990). Data are shown as mean $\mathrm{SEM}$ S. $\mathrm{n}=3$ independent experiments. Statistical analysis was performed using Welch's t-test. ns, not significant. (B) Observation of leaky translation in individual bacteria. The EGFP fluorescence was measured in the absence of IY. In these images, non-specific background fluorescence was completely filtered out (note that this method is distinct from that of Fig. 4A). The exposure time for the EGFP images was twice that in Fig. 3. 

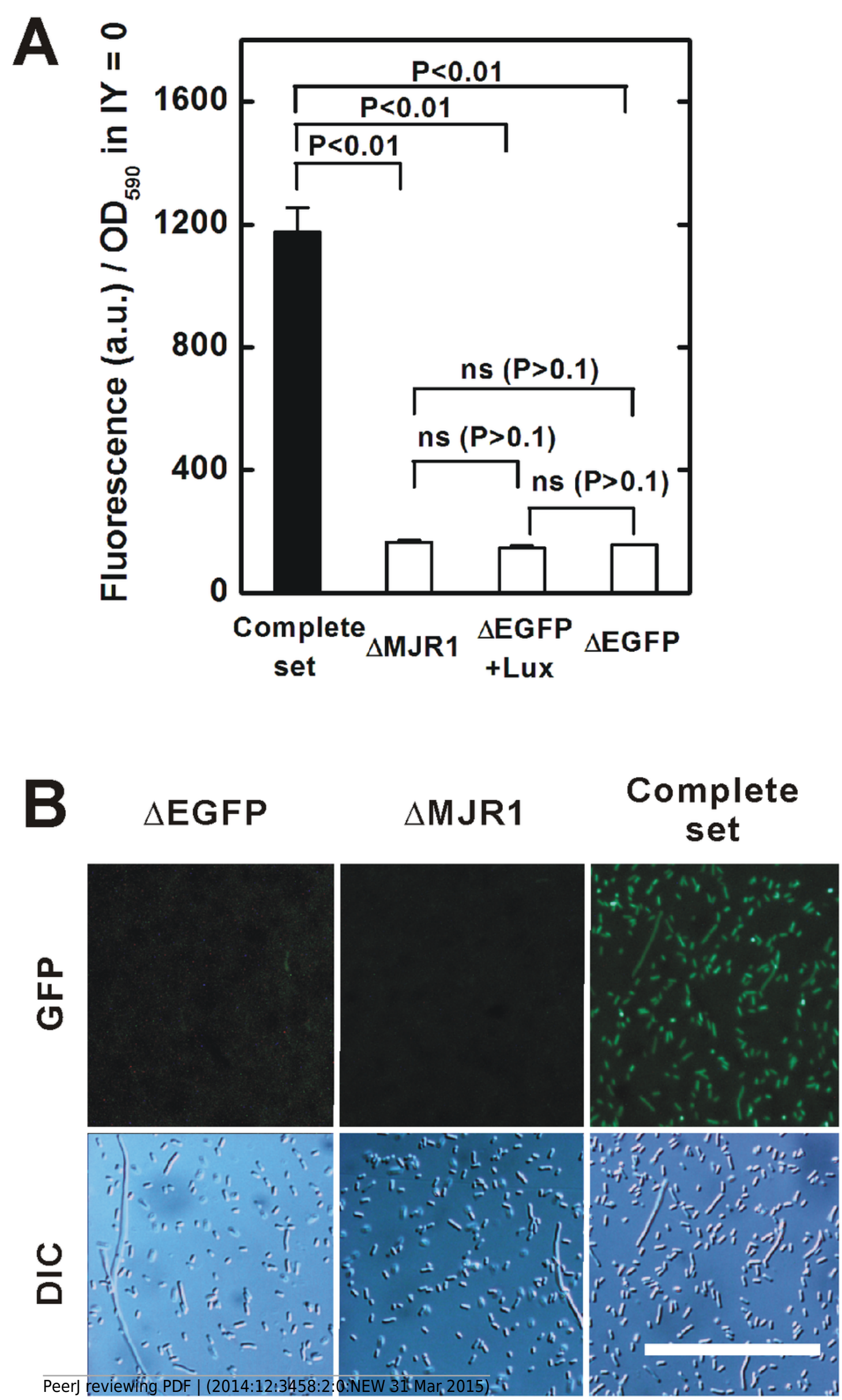